\title{
Anti-androgen receptor ASC-J9 versus anti-androgens MDV3100 (Enzalutamide) or Casodex (Bicalutamide) leads to opposite effects on prostate cancer metastasis via differential modulation of macrophage infiltration and STAT3-CCL2 signaling
}

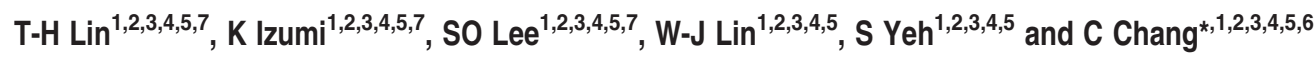

Despite androgen deprivation therapy (ADT) suppression of prostate cancer (PCa) growth, its overall effects on PCa metastasis remain unclear. Using human (C4-2B/THP1) and mouse (TRAMP-C1/RAW264.7) PCa cells-macrophages co-culture systems, we found currently used anti-androgens, MDV3100 (enzalutamide) or Casodex (bicalutamide), promoted macrophage migration to PCa cells that consequently led to enhanced PCa cell invasion. In contrast, the AR degradation enhancer, ASC-J9, suppressed both macrophage migration and subsequent PCa cell invasion. Mechanism dissection showed that Casodex/MDV3100 reduced the AR-mediated PIAS3 expression and enhanced the PSTAT3-CCL2 pathway. Addition of CCR2 antagonist reversed the Casodex/MDV3100-induced macrophage migration and PCa cell invasion. In contrast, ASC-J9 could regulate pSTAT3-CCL2 signaling using two pathways: an AR-dependent pathway via inhibiting PIAS3 expression and an AR-independent pathway via direct inhibition of the STAT3 phosphorylation/activation. These findings were confirmed in the in vivo mouse model with orthotopically injected TRAMP-C1 cells. Together, these results may raise the potential concern about the currently used ADT with anti-androgens that promotes PCa metastasis and may provide some new and better therapeutic strategies using ASC-J9 alone or a combinational therapy that simultaneously targets androgens/AR signaling and PIAS3-pSTAT3-CCL2 signaling to better battle PCa growth and metastasis at castration-resistant stage.

Cell Death and Disease (2013) 4, e764; doi:10.1038/cddis.2013.270; published online 8 August 2013

Subject Category: Cancer

Prostate cancer (PCa) is the leading cause of cancer death in men in the United States. The PCa metastatic lesions are mostly found in the bone, which impair the quality of life and lead to PCa-related deaths in patients. ${ }^{1}$ The androgen deprivation therapy (ADT) is effective in decreasing prostate-specific antigen (PSA) levels and reducing the tumor size with early treatment; however, the effects of ADT on PCa metastasis remain unclear. ${ }^{1}$ Earlier studies in lymph node metastatic tumors showed that the androgen receptor (AR) expression status correlated with patient's prognosis. ${ }^{2,3}$ However, recent findings indicated that AR was differentially correlated with Gleason score patterns being upregulated in Gleason pattern 4 and downregulated in Gleason pattern 5 in both primary and metastatic $\mathrm{PCa} .{ }^{4} \mathrm{~A}$ large clinical statistical report also pointed out that the ADT treatment of patients at earlier stages of $\mathrm{PCa}$ might increase the cancer-specific mortality by promoting more malignant cancer cells. ${ }^{5}$ As the metastasis of cancer is the major cause of cancer-related death, it is important to clarify the AR roles associated with ADT and the differential regulation of tumor growth versus tumor metastases in PCa.

An early report demonstrated that the AR signaling could suppress the wound-healing process by modulating macrophage infiltration with alterations of cytokine expression profiles. $^{6}$ Weitzman and Gordon ${ }^{7}$ found that the gene expression profile in metastatic breast cancer is very similar to the wound-healing process, therefore it is possible that the AR signaling in macrophages might be critical in modulating macrophages infiltration to tumor site and influence $\mathrm{PCa}$ metastasis. Macrophages are the main source of cytokines/chemokines in the local tumor microenvironment ${ }^{8}$ that might be associated with promotion of tumor growth, angiogenesis, and metastasis. ${ }^{9}$ The direct linkage of ADT to cytokines and their detailed mechanisms related to increased $\mathrm{PCa}$ metastasis, however, remain unclear.

\footnotetext{
${ }^{1}$ George Whipple Lab for Cancer Research, University of Rochester Medical Center, Rochester, NY 14642, USA; ${ }^{2}$ Department of Pathology, University of Rochester Medical Center, Rochester, NY 14642, USA; ${ }^{3}$ Department of Urology, University of Rochester Medical Center, Rochester, NY 14642, USA; ${ }^{4}$ Department of Radiation Oncology, University of Rochester Medical Center, Rochester, NY 14642, USA; ${ }^{5}$ The Wilmot Cancer Center, University of Rochester Medical Center, Rochester, NY 14642, USA and ${ }^{6}$ Sex Hormone Research Center, China Medical University and Hospital, Taichung 404, Taiwan

*Corresponding author: Dr C Chang, George Whipple Lab for Cancer Research, University of Rochester Medical Center, 601 Elmwood Avenue, Rochester, NY 14642, USA. Tel: 585273 4500; Fax: 585756 4133; E-mail: chang@urmc.rochester.edu

${ }^{7}$ These authors contributed equally to this work.

Keywords: prostate cancer; metastasis; macrophage; anti-androgens; ASC-J9

Abbreviations: ADT, androgen deprivation therapy; AR, androgen receptor; Anti-A/AR, anti-androgen/AR; PCa, prostate cancer; PIAS3, protein inhibitor of STAT3; PSA, prostate specific antigen; TAM, tumor associated macrophage; PBS, phosphate-buffered saline

Received 22.4.13; revised 12.6.13; accepted 19.6.13; Edited by G Ciliberto
} 
Here we found that ADT with the currently used Casodex (bicalutamide) and the newly developed MDV3100 (enzalutamide) ${ }^{10}$ promoted PCa metastasis via enhancing macrophage infiltration in both in vitro co-culture systems and in vivo mouse models. In contrast, the newly developed AR degradation enhancer ASC-J9 ${ }^{11}$ showed an opposite effect, suppressing the macrophage infiltration and consequent $\mathrm{PCa}$ metastasis. Detailed mechanism studies identified the differential regulation of these three different anti-androgen/AR (anti-A/AR) compounds on STAT3/CCL2 signaling, which explained their opposite effects on macrophage infiltration/ $\mathrm{PCa}$ metastasis. These results may, therefore, provide a potential novel therapy combining Casodex/MDV3100 with anti-CCL2-CCR2 compounds. Alternatively, targeting AR with ASC-J9 to simultaneously suppress PCa growth and macrophage infiltration/metastasis could be a promising future therapy to better battle PCa.

\section{Results}

Differential effects of Casodex/MDV3100 versus ASC-J9 on macrophage migration to PCa cells. We performed in vitro macrophage migration assays using two different $\mathrm{PCa}$ and macrophage cell line sets (human C4-2B/human THP1 cells and mouse TRAMP-C1/mouse RAW264.7 cells) to study ADT effects on PCa cells' capacity to recruit macrophages. We used the two current clinically used anti-androgens, Casodex $(10 \mu \mathrm{M}$, the most widely used anti-androgen in current $\left.\mathrm{ADT}^{12}\right)$ and MDV3100 $(10 \mu \mathrm{M}$, a powerful new anti-androgen that could delay the recurrence of increased PSA with extended $\mathrm{PCa}$ patients survival time to 4.8 months $^{10}$ ), and one newly developed anti-AR compound ASC-J9 (5 $\mu \mathrm{M}$, the newly developed AR degradation enhancer with the unique capability to degrade AR protein in selective cells including $\mathrm{PCa}^{6,11,13,14}$ ).

The PCa C4-2B cells were treated with the three different anti-A/AR compounds for 3 days, and the conditioned media (CM) were collected and applied in the human THP1 macrophage migration assays (Figure 1a). We found the CM from the PCa cells treated with $10 \mu \mathrm{M}$ Casodex or MDV3100 significantly enhanced the macrophage migration (Figure 1b, left panel). Similar results were also obtained when we replaced human C4-2B/THP1 cell set with mouse RAW264.7/ TRAMP-C1 cells set (Figure 1b, right panel). In contrast, we found $5 \mu \mathrm{M}$ ASC-J9 suppressed macrophage recruitment in both human and mouse cell sets (Figure 1b).

Differential effects of Casodex/MDV3100 versus ASC-J9 on $\mathrm{PCa}$ cell invasion under $\mathrm{PCa}$ cells/macrophages co-culture system. The PCa cells/macrophages co-culture system was then used to investigate the consequences of differential recruitment of macrophages to $\mathrm{PCa}$ cells for their impacts on PCa cell invasion. The human C4-2B/THP1 and mouse TRAMP-C1/RAW264.7 cell sets were co-cultured in

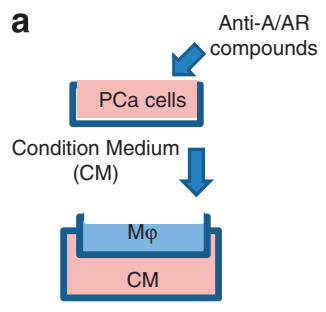

b
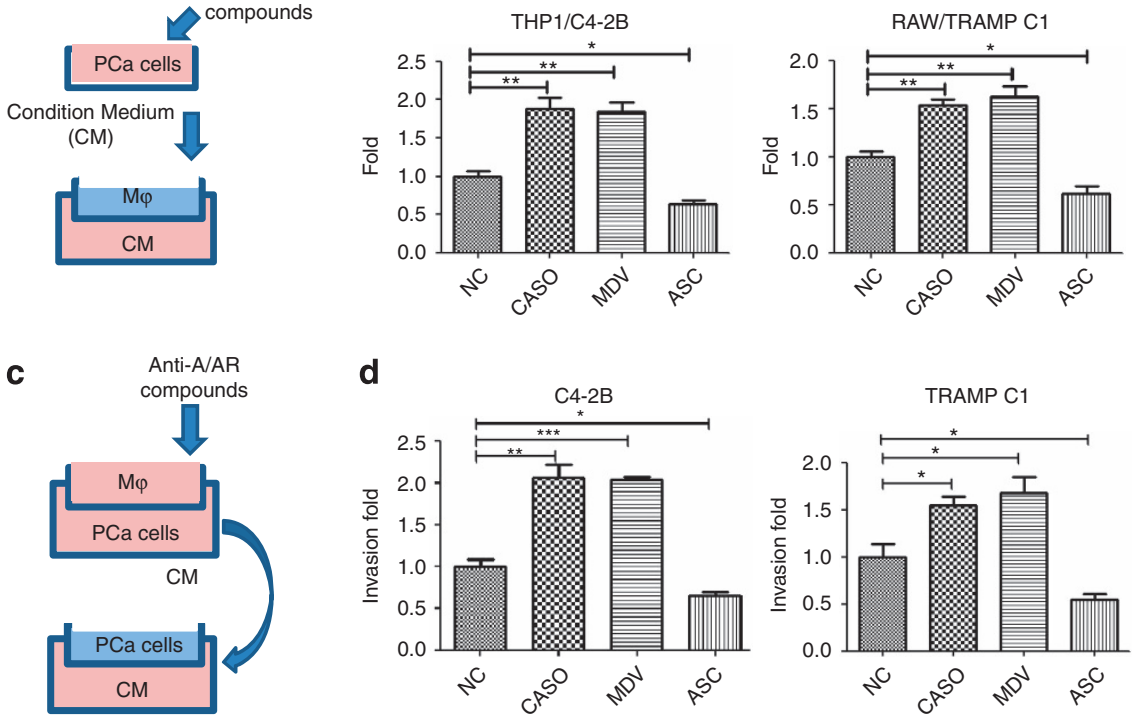

Figure 1 Differential effects of anti-A/AR compounds on macrophage migration and PCa invasion. (a) Illustration of the in vitro macrophage (M $\phi)$ migration model. The PCa cells were treated with anti-A/ARs $10 \mu \mathrm{M}$ Casodex (CASO), $10 \mu \mathrm{M}$ MDV3100 (MDV), $5 \mu \mathrm{M}$ ASC-J9 (ASC), or vehicle control (NC), and incubated in 10\% CD-FBS RPMI medium at $10 \mathrm{nM}$ DHT for 3 days. The CM were collected, diluted with 10\% CD-FBS RPMI medium at 1:1 ratio, and then placed into the lower chamber of transwell plates. (b) Macrophage migration to the PCa cells CM. The THP1 cell migration to the C4-2B cells CM (left) and the RAW264.7 (RAW) cell migration to the TRAMP-C1 cells $\mathrm{CM}$ (right) were shown. (c) Illustration of PCa invasion with macrophage co-culture in vitro model. The PCa cells were plated into lower chambers of the transwell plates, while the macrophage cells were plated in the upper chambers. The co-culture system was treated with different anti-A/AR or vehicle control (NC), and incubated in $10 \%$ CD-FBS RPMI medium at $10 \mathrm{nM}$ DHT for 3 days. The CM were collected, diluted with 10\% CD-FBS RPMI medium at $1: 1$ ratio, and then put into the lower chamber of transwell plates. The parental PCa cells were then placed on the upper chamber of transwell plates with Matrigel pre-coated inserts in serum-free medium for the invasion assay. (d) PCa invasion induced by the co-culture CM. The C4-2B cell invasion with the C4-2B/THP1 cell CM (left) or the TRAMP-C1 cell invasion with the TRAMP-C1/RAW264.7 cell $\mathrm{CM}$ (right) were shown. Error bars $=$ mean \pm S.E.M. ${ }^{\star} P<0.05,{ }^{* \star} P<0.01,{ }^{* * *} P<0.001$ 
the $0.4-\mu \mathrm{m}$ transwell plates with $10 \mu \mathrm{M}$ Casodex, $10 \mu \mathrm{M}$ MDV3100, $5 \mu \mathrm{M}$ ASC-J9 or vehicle control for 3 days. The CM were then collected, diluted, and put into the lower chambers in the transwell plates. The parental $\mathrm{C} 4-2 \mathrm{~B}$ or TRAMP-C1 cells were placed in the upper chambers and invasion assays were performed (Figure 1c). Importantly, we found the $\mathrm{CM}$ from the $\mathrm{PCa} /$ macrophage co-cultured cells treated with $10 \mu \mathrm{M}$ Casodex or MDV3100 significantly enhanced the PCa invasion in both human and mouse cell sets tested (Figure 1d). In contrast, we found $5 \mu \mathrm{M}$ ASC-J9 suppressed PCa invasion in both sets (Figure $1 \mathrm{~d}$ ).

Together, results from Figures 1a-d demonstrated that the Casodex and MDV3100 might promote the macrophage infiltration to PCa cells and then promote PCa cell invasion. In contrast, ASC-J9 could suppress macrophage infiltration to $\mathrm{PCa}$ cells and the consequent PCa cell invasion.

CCR2 antagonist reversed the Casodex/MDV3100induced macrophage infiltration and PCa invasion. The unexpected/unrecognized differential effects of these three different anti-A/AR compounds on macrophage infiltration and their impact on $\mathrm{PCa}$ cell invasion raised very exciting yet important question(s) on how to overcome this unwanted effect of the current ADT with Casodex and MDV3100 increasing macrophage infiltration/PCa cell invasion. We, therefore, determined to further dissect the potential molecular mechanisms behind these contrasting differences.

Among the chemokines that are correlated with macrophage infiltration, the CCL2-CCR2 axis has been shown to have pivotal roles in tumor initiation and progression to metastatic stages. ${ }^{15}$ It was also demonstrated in PCa studies that CCL2 could either directly enhance PCa cell proliferation, survival, and metastasis, ${ }^{15}$ or promote the $\mathrm{PCa}$ progression by indirectly modulating angiogenesis and macrophage recruitment in the tumor microenvironment. ${ }^{15}$ We applied the CCR2 antagonist in the macrophage migration assay to test its capacity to reverse Casodex- and MDV3100-induced macrophage migration. As shown in Figures $2 \mathrm{a}$ and $\mathrm{c}$, addition of $20 \mathrm{nM}$ CCR2 antagonist significantly reversed the macrophages migration in human THP1/C4-2B (Figure 2a) and mouse RAW264.7/TRAMP-C1 (Figure 2c) cells co-culture systems. Furthermore, addition of $20 \mathrm{nM}$ CCR2 antagonist also significantly reversed the macrophages-induced invasion increase in human $\mathrm{C} 4-2 \mathrm{~B}$ (Figure 2b) and mouse TRAMP-C1 cells (Figure 2d). Together, results from Figures $2 a-d$ confirmed that both Casodex and MDV3100 might need to go through CCL2-CCR2 axis to induce macrophage infiltration to $\mathrm{PCa}$ cells, which led to enhanced PCa cell invasion.

The effect of CCR2 antagonist was also investigated on the ASC-J9-suppressed macrophage migration. Importantly, we found $20 \mathrm{nM}$ CCR2 antagonist showed little effect on ASC-J9-suppressed macrophage migration in human THP1/C4-2B (Figure 2a) and mouse RAW264.7/TRAMP-C1 (Figure 2c) cells co-culture systems. Furthermore, 20 nM CCR2 antagonist also showed little effect on ASC-J9-suppressed macrophage-induced invasion increase in human $\mathrm{C} 4-2 \mathrm{~B}$ (Figure 2b) and mouse TRAMP-C1 (Figure 2d) cells, suggesting the ASC-J9 itself might already have similar effects as the CCR2 antagonist to block CCL2-CCR2 axis, so
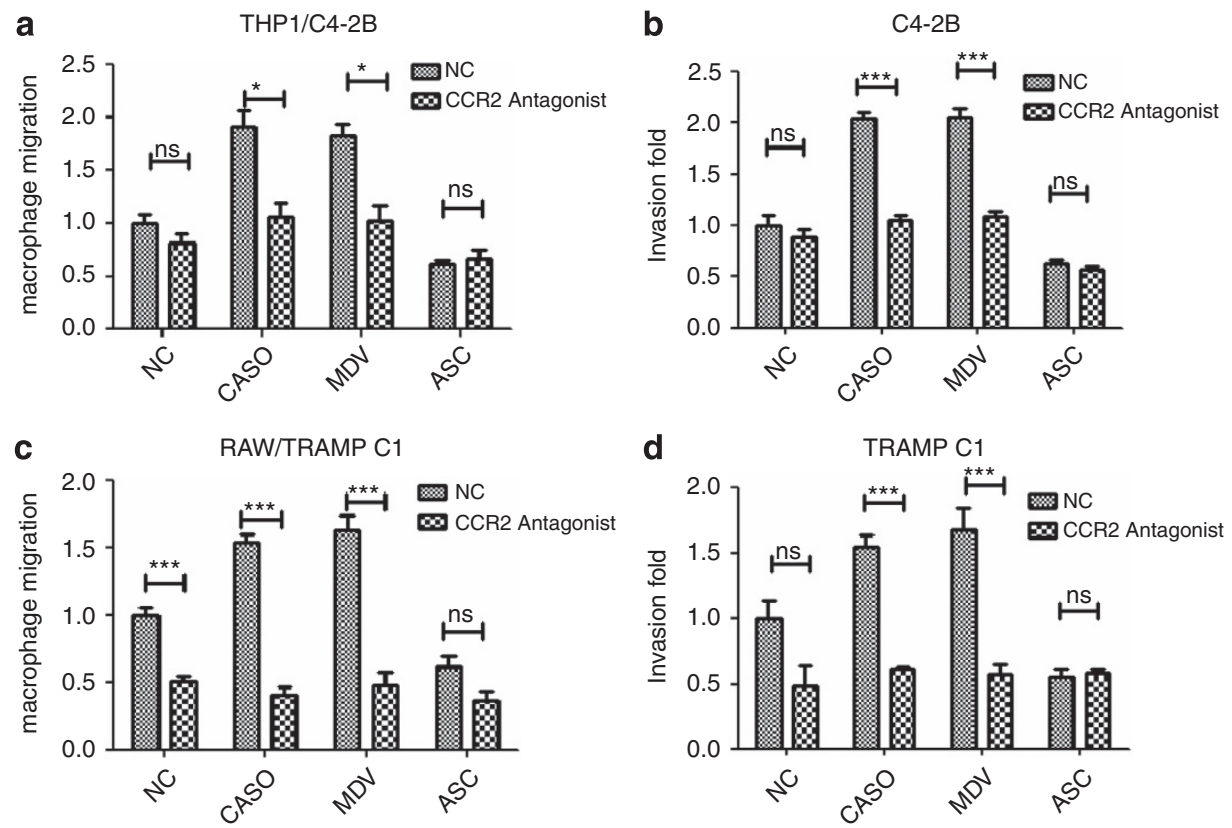

Figure 2 Reversed macrophage migration and PCa invasion by blocking the CCL2/CCR2 interaction. (a and c) The PCa cells were treated with $10 \mu \mathrm{M}$ Casodex (CASO), $10 \mu \mathrm{M}$ MDV3100 (MDV), $5 \mu \mathrm{M}$ ASC-J9, or vehicle control (NC), combined with/without CCR2 antagonist, and incubated in 10\% CD-FBS RPMI medium at $10 \mathrm{nM}$ DHT for 3 days. The CMs were obtained and used for the macrophage migration assays. The THP1 cell migration to the C4-2B cell CM (a) or the RAW264.7 (RAW) cells migration to the TRAMP-C1 cell CM (c) were shown. (b and d) The PCa/macrophage cells co-culture systems were treated with $10 \mu \mathrm{M}$ Casodex (CASO), $10 \mu \mathrm{M}$ MDV3100 (MDV), anti-AR $5 \mu \mathrm{M}$ ASC-J9, or vehicle control, combined with/without CCR2 antagonist, and incubated in 10\% CD-FBS RPMI medium at $10 \mathrm{nM}$ DHT for 3 days. The CMs were obtained and used for the PCa invasion assays. The C4-2B cell invasion with the C4-2B/THP1 cell CM (b) or the TRAMP-C1 cell invasion with the TRAMP-C1/RAW264.7 cells CM (d) was shown. Error bars $=$ mean \pm S.E.M. ${ }^{\star} P<0.05,{ }^{\star *} P<0.01,{ }^{* \star *} P<0.001$. N.S., not significant 
addition of CCR2 antagonist did not further influence ASC-J9 effect.

Together, results from Figures $2 a-d$ clearly demonstrated that ASC-J9 had a unique and better anti-PCa cell invasion capacity than the current two anti-androgens, Casodex and MDV3100, and proved the differential anti-PCa cell invasion effects could be due to opposite modulation of CCL2-CCR2 signaling.

AR-dependent regulation of PIAS3-STAT3-CCL2 signal-
ing and AR-independent regulation of STAT3-CCL2
signaling explain differential modulation of CCL2
signaling by Casodex/MDV3100 (induction) and ASC-J9
(suppression). To further dissect the molecular mechanism by which these three anti-A/AR compounds have differential effects on activation of CCL2-CCR2 signaling, we assayed their influence on CCL2 expression, and as expected, addition of $10 \mu \mathrm{M}$ Casodex or MDV3100 increased CCL2 expression in C4-2B and TRAMP-C1 cells at the mRNA (Figure 3a) and protein (Figure 3b) levels. In contrast, we found $5 \mu \mathrm{M}$ ASC-J9 significantly suppressed the CCL2 expression (and other AR key target genes, including PSA and $\mathrm{Nk} \times 3.1)$ in the $\mathrm{C} 4-2 \mathrm{~B}$ and TRAMP-C1 PCa cells at mRNA (Figure $3 a$ ) and protein (Figure $3 b$ ) levels.

We further examined CCL2 upstream genes STAT3 ${ }^{6}$ and protein inhibitor of activated STAT3 (PIAS3), ${ }^{16}$ as previous studies found that androgen/AR signaling could induce the PIAS3 expression that suppressed the STAT3 activation. ${ }^{17}$

a
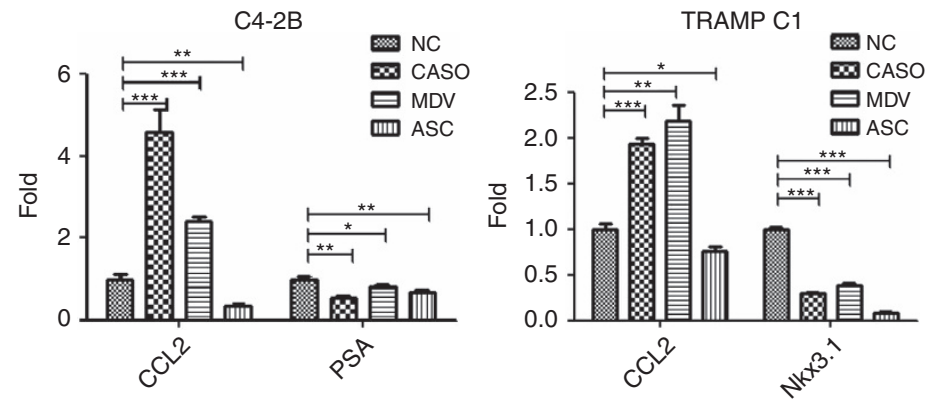

b

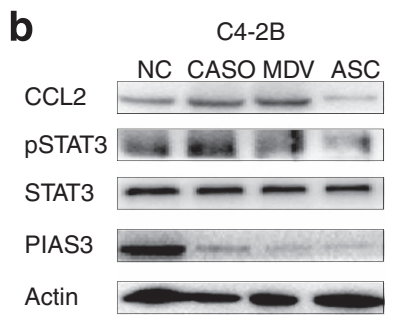

TRAMP C1

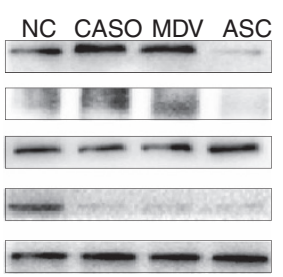

C

PC3
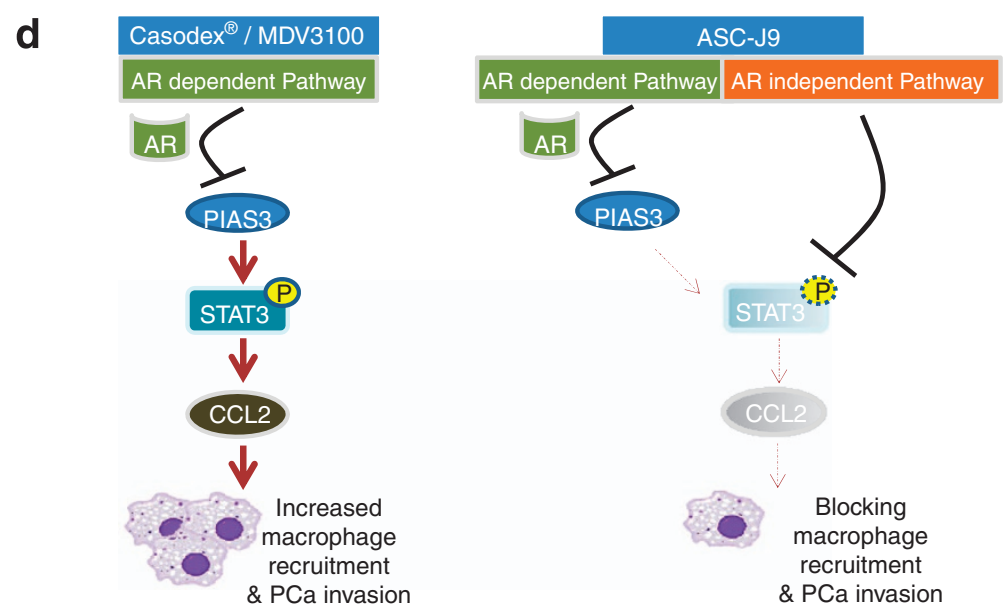

Figure 3 Differential regulation of STAT3/CCL2 pathway by Casodex, MDV3100, and ASC-J9. (a) Casodex and MDV3100 enhanced, but ASC-J9 suppressed CCL2 expression. The C4-2B and TRAMP-C1 cells were treated with $10 \mu \mathrm{M}$ Casodex (CASO), $10 \mu \mathrm{M}$ MDV3100 (MDV), $5 \mu \mathrm{M}$ ASC-J9 (ASC), or vehicle control (NC), incubated in $10 \%$ CD-FBS RPMI media at $10 \mathrm{nM}$ DHT concentration for $24 \mathrm{~h}$, and the gene expression was determined by qPCR. (b) Casodex and MDV3100 enhanced, but ASC-J9 suppressed, STAT3 activation. The effects of three different anti-A/AR compounds on the expression of CCL2, PIAS3, pSTAT3, STAT3, and GAPDH in C4-2B and TRAMP-C1 cells were analyzed by western blotting. (c) ASC-J9 inhibited STAT3/CCL2 signaling via AR-independent pathway. The effects of three different anti-A/AR compounds on the expression of CCL2, PIAS3, pSTAT3, STAT3, and GAPDH in AR-negative PC3 cells were analyzed by western blotting. (d) Illustration of AR-dependent and AR-independent pathway of STAT3/CCL2 regulation by ASC-J9. Error bars $=$ mean \pm S.E.M., ${ }^{*} P<0.05,{ }^{* \star} P<0.01,{ }^{* \star *} P<0.001$. N.S., not significant 
The results showed that addition of $10 \mu \mathrm{M}$ Casodex or MDV3100 suppressed PIAS with enhanced STAT3 activation in C4-2B and TRAMP-C1 cells (Figure 3b).

Importantly, although ASC-J9 was able to suppress PIAS3 expression, we could not observe consequent enhanced STAT3 phosphorylation/activation in C4-2B and TRAMP-C1 cells and in contrast, suppressive effects were observed (Figure 3b), as well as suppressed CCL2 expression. These unexpected findings led us to believe that in addition to the ASC-J9 effect on AR-dependent modulation of PIAS3pSTAT3-CCL2 signaling, ASC-J9 might be able to go through another unique AR-independent signaling pathway to suppress pSTAT-CCL2 signaling. We then tested this hypothesis in PCa AR-negative PC3 cells, and found that both $10 \mu \mathrm{M}$ Casodex or MDV3100 treatment had little effect on the PIAS3-STAT3/CCL2 signaling, confirming their antiandrogen effects are AR-dependent. As expected, we found $5 \mu \mathrm{M}$ ASC-J9 treatment resulted in significant suppression of the STAT3 phosphorylation/activation and CCL2 expression, with little influence on the PIAS3 expression in the ARnegative PC3 cells (Figure 3c), confirming ASC-J9 could also go through an AR-independent pathway to modulate STAT3 phosphorylation/activation and CCL2 expression.

Together, results from Figures $3 a-c$ clearly proved that ASC-J9 could go through an AR-dependent mechanism to alter PIAS3-pSTAT3-CCL2 signaling that induced CCL2 expression as well as go through an AR-independent mechanism to alter pSTAT3-CCL2 signaling that suppressed CCL2 expression. The consequences of these two opposite effects result in suppression of CCL2 expression, which indicates that the AR-independent pathway may be more dominant than the AR-dependent pathway (Figure 3d).

This unique way of modulation of CCL2 expression suggests that ASC-J9 is the first identified anti-AR compound that not only has anti-PCa cells proliferation capacity, more importantly, may also have anti-PCa cell invasion capacity. In contrast, the currently used anti-androgens, such as Casodex or MDV3100, may have the anti-PCa cells proliferation capacity, but have the capacity of enhancing PCa cell invasion undesirably. To our best knowledge, ASC-J9 can have dual capacities that could suppress both $\mathrm{PCa}$ cell growth and $\mathrm{PCa}$ cell invasion via AR-dependent and AR-independent mechanisms.

ASC-J9, but not Casodex and MDV3100, suppressed both macrophage infiltration and PCa metastasis in the in vivo mouse models. To further prove the opposite effects of these anti-A/AR compounds on macrophage migration and $\mathrm{PCa}$ cell invasion in vivo, we used mouse models with orthotopically xenografted TRAMP-C1 cells, as this model is well established to study the $\mathrm{PCa}$ metastasis in vivo. ${ }^{18} \mathrm{~A}$ total of $10^{6}$ TRAMP-C1 cells were mixed with Matrigel and injected into the anterior prostates of nude mice. After 2 weeks, the three different anti-A/AR compounds (Casodex at $30 \mathrm{mg} / \mathrm{kg}$; MDV3100 at $30 \mathrm{mg} / \mathrm{kg}$, and ASC-J9 at $75 \mathrm{mg} / \mathrm{kg}$ ) were i.p. injected every other day for 3 weeks (Figure 4a). After 3 weeks of treatment, the mice were killed and the visible metastatic tumors were carefully evaluated.

We found the Casodex- and MDV3100-treated mice had significant increase (Figure 4b, left), yet the ASC-J9 treated mice had significant decrease (Figure 4b, left) in developing distant metastatic tumors in diaphragm and lymph nodes, compared with the vehicle control mice (Figure 4b, left). Histological examination of metastatic tumor tissues confirmed metastases originated from the TRAMP-C1inoculated cells (Figure 4b, middle and right).

Importantly, we found little change in mice body weight among all the mice treated (Figure 4c), and early reports ${ }^{19-22}$ also suggested that mice treated with ASC-J9 had little side effects or toxicity.

Combination treatment of ASC-J9 plus CCR2 antagonist in TRAMP-C1-implanted mice did not further reduce the metastases when compared with the ASC-J9-treated-mice (Figure 4d), which confirmed our in vitro data described in Figures 2a-d and also proved that ASC-J9, by itself, has antiCCL2-CCR2 capacity and therefore CCR2 antagonist action was not necessary to suppress the CCL2-CCR2 signaling.

The macrophage infiltration and the activation of individual molecules in STAT3-CCL2 signaling in tumor tissues of the mice treated with different anti-A/AR compounds were also evaluated by the immunohistochemistry staining. As shown in Figure $4 \mathrm{e}$, we found the macrophage infiltration (the number of $\mathrm{F} 4 / 80$ positively stained cells) was increased in Casodex- or MDV3100-treated tumors, whereas the ASC-J9-treated tumors had fewer infiltrated macrophages. On the other hand, while the expression of AR target gene Nkx3.1 was inhibited by all anti-A/AR compounds treatments, expressions of STAT3 and CCL2 were higher in Casodex- or MDV3100treated tumor samples, but lower in ASC-J9-treated tumor samples, when compared with the tumor samples from the vehicle control group (Figure 4f), all of which were in agreement with our in vitro results described in Figure 3.

Together, results from in vivo TRAMP-C1 mouse model studies confirmed our in vitro cell line studies and demonstrated that ADT with Casodex and MDV3100 treatment might promote macrophage infiltration and enhance PCa metastasis, yet ASC-J9 treatment could suppress macrophage infiltration and PCa metastasis, and these differential effects involved the opposite regulation of STAT3-CCL2 signaling.

\section{Discussion}

While ADT can effectively inhibit the androgen/AR-mediated $\mathrm{PCa}$ cell growth, the roles of ADT in PCa metastasis remain unclear and early studies found expression of several metastatis-related markers, such as $\mathrm{N}$-cadherin, ${ }^{23}$ Cadherin-11, ${ }^{24,25}$ and nestin, ${ }^{26}$ were increased in human clinical PCa samples after ADT, suggesting that ADT targeting of androgen/AR signaling may promote $\mathrm{PCa}$ metastasis. The animal studies using the TRAMP mouse model also demonstrated that the mice with ADT using surgical castration (so that their serum testosterone is undetectable) had increased lymph node ${ }^{27}$ or distant ${ }^{28}$ metastases. Furthermore, Niu et al. ${ }^{29}$ found in the TRAMP mouse model studies that the mice with AR knockdown in prostate epithelial cells had increased metastatic PCa with earlier deaths.

The above human and mice data showing ADT might enhance PCa metastasis were also in agreement with in vivo/ in vitro data using various PCa cell lines, for example, 
a

Tumor implantation (Week 0)

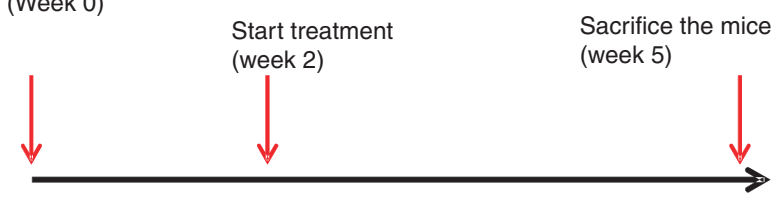

\section{$\begin{array}{ll}\text { Treatment Dose (3 times/week) } & \text { D }\end{array}$}

Vehicle control

Casodex $\quad 30 \mathrm{mg} / \mathrm{kg}$

MDV3100 $30 \mathrm{mg} / \mathrm{kg}$

ASC-J9 ${ }^{\circledR} \quad 75 \mathrm{mg} / \mathrm{kg}$

b Metastasis

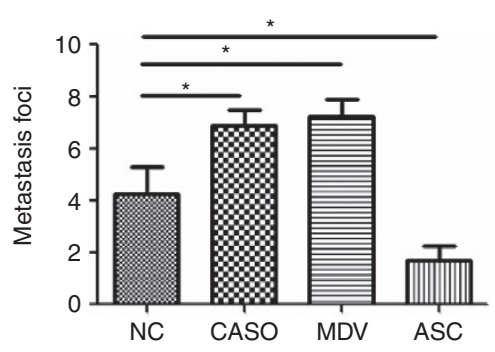

C
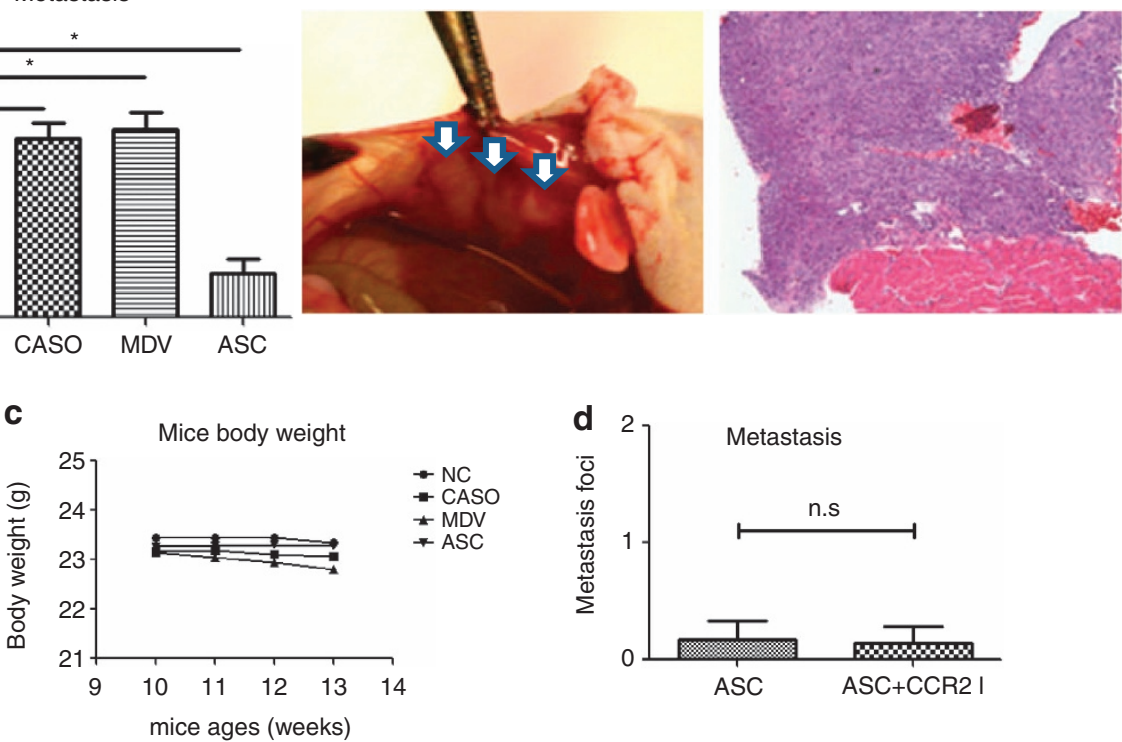

mice ages (weeks)

e

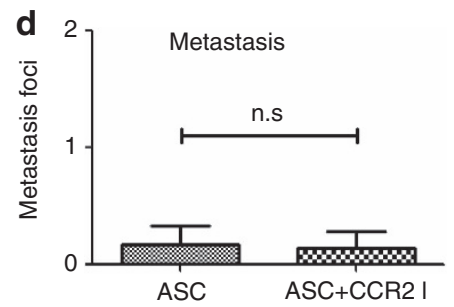

NC

CASO

MDV
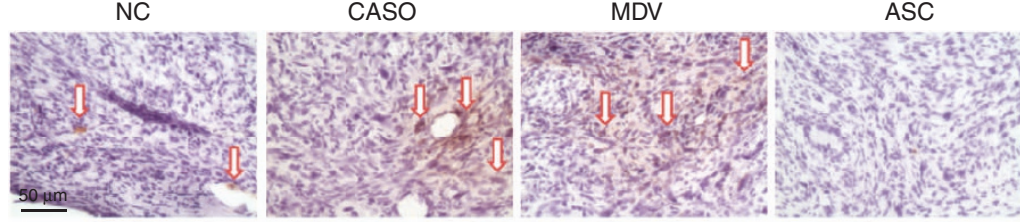

CASO

f

pSTAT3

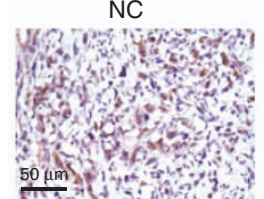

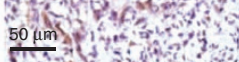

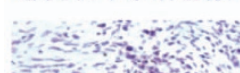

CCL2
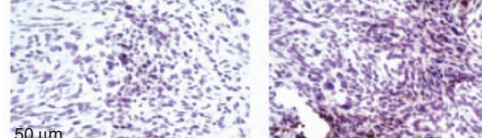

$50 \mathrm{~nm}=\mathrm{w}, \mathrm{s}$
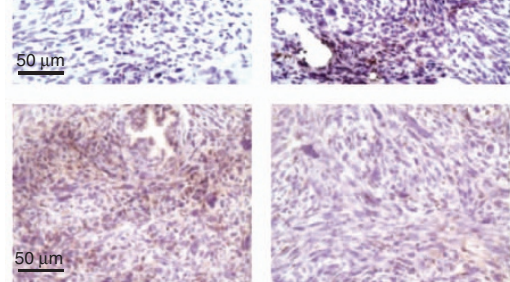
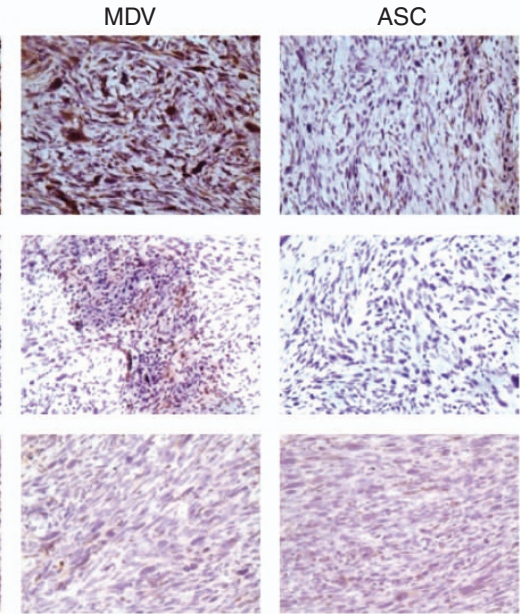

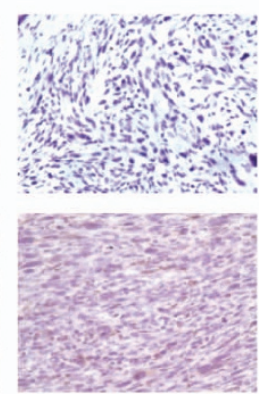

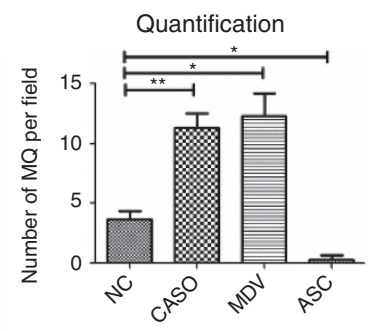
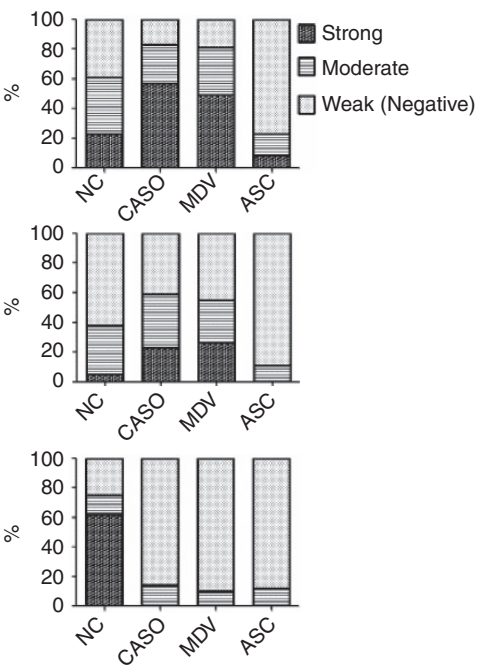
castration in mice with orthotopically xenografted PCa C4-2 cells led to increased metastases, yet the detailed mechanism remains unclear. ${ }^{30} \mathrm{~A}$ recent report also showed that ADT could increase the EMT process in a ZEB1-dependent manner in human LuCaP35 PCa cell xenograft mice model. ${ }^{31}$

Furthermore, a recent clinical trial of ADT with abiraterone, which suppressed androgen biosynthesis, found that while $79 \%$ of $\mathrm{PCa}$ patients have a decline of $50 \%$ or more in their PSA level, yet $52 \%$ of these PCa patients have either enhanced bone lesions or increased new bone lesions after 4 months of treatment, and these increases were observed at stages when patients' PSA dropped to very low levels. ${ }^{32}$ Meanwhile, the enhanced bone lesions were reduced after 7 months of treatment, suggesting that abiraterone may still be capable to control the PCa growth in metastatic sites.

Importantly, recent studies also found the current ADT with anti-androgens might lead to primary tumor reduction with PSA decreased yet metastases increased in some $\mathrm{PCa}$ patients. ${ }^{33}$ In vitro results from four different $\mathrm{PCa}$ cell lines also showed that $10 \mu \mathrm{M}$ Casodex or MDV3100 treatments suppressed $\mathrm{PCa}$ cell growth and reduce PSA level, yet significantly enhanced PCa cell invasion. In vivo mice studies using orthotopic xenograft mouse model also confirmed these results. In contrast, ASC-J9 led to suppressed PCa cell growth and cell invasion in in vitro and in vivo models. Mechanism dissection indicated these Casodex/MDV3100 treatments enhanced the TGF- $\beta 1 /$ Smad3/MMP9 pathway, but ASC-J9 showed promising anti-invasion effects via downregulation of MMP9 expression. ${ }^{33}$

Together with our current findings, the clinical evidence and animal experimental results suggested that anti-A/AR signaling involved in $\mathrm{PCa}$ proliferation versus metastasis could be via two unrelated pathways, and a new combination therapy to suppress PCa proliferation with the current ADT plus a new therapy to battle metastasis may be developed in the future.

The infiltration of tumor-associated macrophages (TAM) has been linked to tumor progression in many tumor diseases, suggesting the potential of TAM-targeting therapy in the treatment of advanced cancer. ${ }^{34}$ It has been shown that TAM can provide growth and survival factors, induce angiogenesis, and enhance matrix remodeling in the tumor microenvironment, consequently leading to tumor metastases. CCL2 is highly expressed in malignant tumor cells, and may have an essential role in TAM recruitment. ${ }^{15}$ After recruitment, the TAM can also produce CCL2, suggesting a positive feedback loop may exist in the cross-talk between PCa and TAM. ${ }^{35}$ Our data indicated that ADT could induce the CCL2 expression and macrophage infiltration, whereas the CCR2 antagonist can reverse the ADT-induced tumor malignancy. These results implied that CCL2 might have critical roles in ADTinduced PCa metastasis. While the use of the CCL2 antibody failed in a phase II clinical trial, ${ }^{36}$ CCR2 antagonist may be an alternative approach to manage the side effects caused by ADT. Interestingly, the use of the ASC-J9 alone can suppress, but not induce, the CCL2 expression, highlighting its potential in clinical application.

Previously, we have demonstrated the unique capability of ASC-J9 in AR protein degradation in selective cell types. Interestingly, our current results identified the new characteristics of ASC-J9 in STAT3 inhibition in an AR-independent manner. The STATs are transcription factors that have important roles during the inflammation process. Among them, STAT3 is highly expressed and may link to cancer progression in many cancer types including PCa. Activation of STAT3 is responsible for many genes involved in tumor malignancy including CCL2, making it a valuable target in cancer therapy. The inhibition of STAT3 can be achieved by indirect targeting of the upstream tyrosine kinase JAK1/2, or direct targeting of the STAT3 by oligonucleotides or siRNA. ${ }^{37}$ As the total STAT3 protein level was not influenced by ASC-J9, it would be interesting to further investigate whether ASC-J9 can inhibit the STAT3 activation through blocking the upstream JAK1/2 kinase activity. When applied in the PCa studies, ASC-J9 can inhibit the PIAS3 gene expression in AR-positive cells, which may result in STAT3 activation. On the other hand, it can also block the STAT3 phosphorylation/activation in both AR-positive and AR-negative cells. The consequences of both AR-dependent STAT3 activation and AR-independent anti-STAT3 activation by ASC-J9 then leads to the suppression of CCL2 expression in PCa cells, which may then result in blocking of PCa invasion.

In conclusion, our current data suggest that the current clinically used ADT with Casodex/MDV3100 in PCa patients might lead to unwanted side effects of enhanced macrophage infiltration/PCa metastasis through modulation of CCL2STAT3 signaling. The combination of ADT with anti-STAT3 or anti-CCL2-CCR2 compounds, or the use of the novel AR degradation enhancer, ASC-J9, may help to prevent the unwanted side effects induced by ADT, which may help us to develop new therapeutic approaches in the future to better battle PCa at the castration-resistant stage.

\section{Materials and Methods}

Cell culture. The C4-2B cell line, which was derived from a bone metastasis in the castrated LNCaP xenografted mouse, was a kind gift from Dr. Ming Jiang. TRAMP-C1, THP1, and RAW264.7 cell lines were obtained from ATCC (Manasses, VA, USA). The C4-2B and TRAMP-C1 cell lines were maintained in RPMI-1640 medium containing 10\% non-heat-inactivated fetal bovine serum

Figure 4 Induction versus suppression of PCa metastases by Casodex, MDV3100, and ASC-J9. (a) Outline of the in vivo experiment. The TRAMP-C1 cells were used as in vivo mouse model to detect the tumor metastases in the transplanted nude mice. (b) Quantification of metastatic lesions in mice treated with different anti-A/AR compounds. After euthanizing the mice, the metastatic tumors were evaluated, and the numbers of metastatic foci in each mouse were quantified (left). The metastatic tumors observed on the diaphragm (middle) were confirmed by histology with HE staining (right). (c) Body weights of mice under anti-A/AR compounds treatment. The mice body weights were checked weekly starting from the first injection of compounds. (d) Quantification of metastatic lesions in mice treated with ASC-J9 alone or combined with CCR2 antagonist. (e) Migration of macrophages (arrows) into the primary tumor site. The paraffin-embedded tumor tissue sections were stained using antibody of macrophage marker F4/80 (left). The total numbers of macrophages $(M \phi)$ in three randomly selected fields were quantified on the right. (f) Comparisons of STAT3/CCL2 signal in in vivo PCa tissue. The PSTAT3, CCL2, and Nkx3.1 expressions were detected by immunohistochemistry staining of the paraffin-embedded tissue sections $(\times 400)$, the quantification results were shown on right. Error bars $=$ mean \pm S.E.M. ${ }^{\star} P<0.05,{ }^{* \star} P<0.01$. N.S., not significant 
(FBS) and antibiotics (100 units/ml penicillin, $100 \mu \mathrm{g} / \mathrm{ml}$ streptomycin). The THP1 and RAW264.7 cells were maintained in the RPMI medium containing $10 \%$ heatinactivated FBS, antibiotics, and $0.05 \mathrm{mM}$ 2-mercaptoethanol. Cells were cultured at $5 \% \mathrm{CO}_{2}$ and $37^{\circ} \mathrm{C}$.

Reagents. Casodex was purchased from Toronto Research Chemicals (Toronto, ON, Canada). MDV3100 for in vitro studies was purchased from Selleck Chemicals (Houston, TX, USA). CCR2 antagonist was purchased from Santa Cruz Biotechnology (Santa Cruz, CA, USA). ASC-J9 and the MDV3100 used for in vivo studies were synthesized by Dr. Defeng Xu in Shanghai Jiao Tong University. All other chemicals and solvents used in this study were of reagent grade or high performance liquid chromatography grade.

Macrophage recruitment assay. PCa cells were treated with the antiandrogens, together with/without the inhibitors for 3 days. The CM or control media were collected, diluted with $10 \%$ charcoal-dextran-treated (CD)-FBS RPMI media, plated into the lower chamber of transwell plates with a $5-\mu \mathrm{m}$ pore polycarbonate membrane insert (Corning, Corning, NY, USA). THP1 $\left(1 \times 10^{5}\right)$ or RAW264.7 $\left(2 \times 10^{4}\right)$ cells were plated onto the upper chamber for macrophage migration assay. The cells that migrated into the lower chamber media were collected and counted by the Bio-Rad (Hercules, CA, USA) TC10 automatic cell counter. Each sample was assayed in triplicate and the experiments were repeated twice.

Cell invasion assay. $\mathrm{PCa} /$ macrophage cells were co-cultured in $0.4-\mu \mathrm{m}$ transwell plates. Cells were treated with the anti-A/AR drugs for 3 days, with/without the inhibitors. The $\mathrm{CM}$ or control media were collected, diluted with $10 \%$ CD-FBS RPMI media, and plated into the lower chambers of the $6.5-\mathrm{mm}$ transwells with 8- $\mu \mathrm{m}$ pore polycarbonate membrane insert (Corning) pre-coated with $20 \%$ matrigel for cell invasion assay. The parental PCa cells $\left(1 \times 10^{5}\right)$ without treatment were plated onto upper chambers. Each sample was assayed in triplicate. The cells invaded into the lower chamber were fixed, stained using $1 \%$ toluidine blue, and six randomly selected were counted. Each experiment was repeated at least twice.

Quantitative PCR. Total RNAs were extracted from PCa cells using Trizol (Invitrogen, Carlsbad, CA, USA). Reverse transcription was performed using the iScript reverse transcription kit (Bio-Rad). Quantitative real-time PCR analyses using the comparative CT method were performed on a Bio-Rad CFX96 Sequence Detector System using the SYBR Green PCR Master Mix kit (Perkin Elmer, Applied Biosystems, Wellesley, MA, USA) according to the manufacturer's instructions. After an initial incubation at $50^{\circ} \mathrm{C}$ for $2 \mathrm{~min}$ and $10 \mathrm{~min}$ at $95^{\circ} \mathrm{C}$, amplification was performed for 40 cycles at $95^{\circ} \mathrm{C}$ for $20 \mathrm{~s}, 65^{\circ} \mathrm{C}$ for $20 \mathrm{~s}$, and $72{ }^{\circ} \mathrm{C}$ for $30 \mathrm{~s}$. Specific primer pairs were determined with the Primer-Express program (Applied Biosystems, Foster City, CA, USA). The PSA primer pairs were $5^{\prime}$-AGGCCTTCCCTGTACACCAA-3' and $5^{\prime}$-GTCTTGGCCTGGTCATTTCC-3'. The mouse NKX3.1 primer pairs were $5^{\prime}$-CCGGAGGACCCACCAAGTAT- $3^{\prime}$ and $5^{\prime}$-CCTGGATTATGTTCACAGTCCAA-3'. The human CCL2 primer pairs were $5^{\prime}$-CAGCCAGATGCAATCAATGCC- $3^{\prime}$ and $5^{\prime}$-TGGAATCCTGAACCCACTTCT-3'. The mouse CCL2 primer pairs were $5^{\prime}$-TAAAAACCTGGATCGGAACCAAA- $3^{\prime}$ and $5^{\prime}$-GCATTAGCTTCAGATTTACGGGT-3' The normalization control used was $\beta$-actin, $5^{\prime}$-TCACCCACACTGTGCCCCATCTACGA-3' and $5^{\prime}$-CAGCGGAACCGC TCATTGCCAATGG-3' for human, and $5^{\prime}$-GTGACGTTGACATCCGTAAAGA-3' and $5^{\prime}$-GCCGGACTCATCGTACTCC-3' for mouse.

Western blot. Cells were washed with $1 \times$ PBS and scraped into a lysis buffer containing the proteinase inhibitor cocktail (Roche, Nutley, NJ, USA). Protein concentrations were measured with the BCA protein reagent (Pierce Chemical, Rockford, IL, USA). Approximately $50 \mu \mathrm{g}$ of protein/lane were loaded and run on the polyacrylamide gel with a Tris/glycine running buffer system and then transferred onto a PVDF membrane. The antibodies, PIAS3 from Abcam (Cambridge, UK), STAT3 and pSTAT3 from Cell Signaling (Danvers, MA, USA), CCL2 from Sigma (St. Louis, MO, USA), Actin from Santa Cruz Biotechnology with dilutions of $1: 500$ to $1: 1000$ were incubated overnight in the cold room with vigorous shaking. The horseradish peroxidase-conjugated secondary antibody (Pierce Chemical) was used and the signals were detected by adding the enhanced chemiluminescence western blotting detection reagents (Amersham Biosciences, Piscataway, NJ, USA). The quantification of western blotting results was done by Image Lab statistic software (Bio-Rad).
Orthotopic-injected mice model. Male 6- to 8-week-old nude mice were purchased from $\mathrm{NCl}$ (Bethesda, MD, USA). The Matrigel mixture with $1 \times 10^{6}$ TRAMP-C1 cells were orthotopically injected into both anterior prostates. When the tumors were palpable after 2 weeks of implantation, the mice were randomly assigned into four experimental groups and treated with either vehicle, $30 \mathrm{mg} / \mathrm{kg}$ Casodex, $30 \mathrm{mg} / \mathrm{kg}$ MDV3100, or $75 \mathrm{mg} / \mathrm{kg}$ ASC-J9, by i.p. injection three times per week for 3 weeks. The mouse body weights were monitored weekly during the 3 weeks of treatment. After killing, the primary and metastatic (regional and distal) tumor volumes were measured. The tissue samples were then fixed and processed as paraffin tissue sections, the tumor lesions were confirmed by $\mathrm{HE}$ staining.

Immunohistochemistry. Tumor tissues were fixed in $4 \%$ neutral buffered para-formaldehyde and embedded in paraffin. The primary antibodies of the rabbit anti-pSTAT3 (Cell Signaling), the rabbit anti-CCL2 (eBioscience, San Diego, CA, USA) and the rabbit anti-F4/80 (Abcam, Cambridge, UK) were used for staining. The primary antibody was recognized by the biotinylated secondary antibody (Vector, Burlingame, CA, USA), and visualized by VECTASTAIN ABC peroxidase system and peroxidase substrate DAB kit (Vector). The positive staining signals were semiquantitated by ImageJ software.

Statistics. Data are presented as the means \pm S.E.M. for the indicated number of separate experiments. The statistical significance of differences between two groups of data was analyzed by paired $t$-test or Fisher's exact test and $P$-values $<0.05$ were considered significant. In the in vivo animal experiments, measurements of tumor volume and body weights among the four groups were analyzed through one-way ANOVA coupled with the Newman-Keuls test.

\section{Conflict of Interest}

ASC-J9 was patented by the University of Rochester, University of North Carolina, and AndroScience, and then licensed to AndroScience. Both the University of Rochester and $C$ Chang own royalties and equity in AndroScience. The remaining authors declare no conflict of interest.

Acknowledgements. We thank Karen Wolf for help in the preparation of this manuscript. This work was supported by NIH grant CA156700 and George Whipple Professorship Endowment, and Taiwan Department of Health Clinical Trial and Research Center of Excellence grant DOH99-TD-B-111-004, and China 973 National Program on Key Basic Research Project (no. 2012CB518305).

\section{Author Contributions}

T-HL and $\mathrm{Kl}$ performed experiments and analyzed the data; T-HL and SOL wrote the manuscript; SOL and W-JL designed the experiments. SYY and CSC supervised the whole study and participated in the discussion.

1. Miyamoto $\mathrm{H}$, Messing EM, Chang $\mathrm{C}$. Androgen deprivation therapy for prostate cancer: current status and future prospects. Prostate 2004; 61: 332-353.

2. Donovan MJ, Osman I, Khan FM, Vengrenyuk Y, Capodieci P, Koscuiszka M et al. Androgen receptor expression is associated with prostate cancer-specific survival in castrate patients with metastatic disease. BJU Int 2009; 105: 462-467.

3. Sweat SD, Pacelli A, Bergstralh EJ, Slezak JM, Cheng L, Bostwick DG. Androgen receptor expression in prostate cancer lymph node metastases is predictive of outcome after surgery. J Urol 1999; 161: 1233-1237.

4. Fleischmann A, Rocha C, Schobinger S, Seiler R, Wiese B, Thalmann GN. Androgen receptors are differentially expressed in Gleason patterns of prostate cancer and down-regulated in matched lymph node metastases. Prostate 2010; 71: 453-460.

5. Lu-Yao GL, Albertsen PC, Moore DF, Shih W, Lin Y, DiPaola RS et al. Survival following primary androgen deprivation therapy among men with localized prostate cancer. JAMA 2008; 300: 173-181.

6. Lai JJ, Lai KP, Chuang KH, Chang P, Yu IC, Lin WJ et al. Monocyte/macrophage androgen receptor suppresses cutaneous wound healing in mice by enhancing local TNF-alpha expression. J Clin Invest 2009; 119: 3739-3751.

7. Weitzman SA, Gordon LI. Inflammation and cancer: role of phagocyte-generated oxidants in carcinogenesis. Blood 1990; 76: 655-663.

8. Loberg RD, Ying C, Craig M, Yan L, Snyder LA, Pienta KJ. CCL2 as an important mediator of prostate cancer growth in vivo through the regulation of macrophage infiltration. Neoplasia 2007; 9: 556-562. 
9. Talmadge JE, Donkor M, Scholar E. Inflammatory cell infiltration of tumors: Jekyll or Hyde. Cancer Metastasis Rev 2007; 26: 373-400.

10. Scher HI, Beer TM, Higano CS, Anand A, Taplin ME, Efstathiou E et al. Antitumour activity of MDV3100 in castration-resistant prostate cancer: a phase 1-2 study. Lancet 2010; 375. $1437-1446$

11. Lai KP, Huang CK, Chang YJ, Chung CY, Yamashita S, Li L et al. New therapeutic approach to suppress castration-resistant prostate cancer using ASC-J9 via targeting androgen receptor in selective prostate cells. Am J Pathol 2012; 182: 460-473.

12. Tyrrell CJ, Kaisary AV, Iversen $P$, Anderson JB, Baert L, Tammela T et al. A randomised comparison of 'Casodex' (bicalutamide) $150 \mathrm{mg}$ monotherapy versus castration in the treatment of metastatic and locally advanced prostate cancer. Eur Urol 1998; 33: 447-456.

13. Wu MH, Ma WL, Hsu CL, Chen YL, Ou JH, Ryan CK et al. Androgen receptor promotes hepatitis $B$ virus-induced hepatocarcinogenesis through modulation of hepatitis $B$ virus RNA transcription. Sci Transl Med 2010; 2: 32ra5.

14. Yang Z, Chang YJ, Yu IC, Yeh S, Wu CC, Miyamoto H et al. ASC-J9 ameliorates spinal and bulbar muscular atrophy phenotype via degradation of androgen receptor. Nat Med 2007 13: 348-353.

15. Zhang J, Patel L, Pienta KJCC. chemokine ligand 2 (CCL2) promotes prostate cancer tumorigenesis and metastasis. Cytokine Growth Factor Rev 2009; 21: 41-48.

16. Chung CD, Liao J, Liu B, Rao X, Jay P, Berta P et al. Specific inhibition of Stat3 signal transduction by PIAS3. Science 1997; 278: 1803-1805.

17. Junicho A, Matsuda T, Yamamoto T, Kishi H, Korkmaz K, Saatcioglu F et al. Protein inhibitor of activated STAT3 regulates androgen receptor signaling in prostate carcinoma cells. Biochem Biophys Res Commun 2000; 278: 9-13.

18. Somers KD, Brown RR, Holterman DA, Yousefieh N, Glass WF, Wright GL Jr. et al. Orthotopic treatment model of prostate cancer and metastasis in the immunocompetent mouse: efficacy of flt3 ligand immunotherapy. Int J Cancer 2003; 107: 773-780.

19. Kyprianou N, Isaacs JT. Expression of transforming growth factor-beta in the rat ventral prostate during castration-induced programmed cell death. Mol Endocrinol 1989; 3 : 1515-1522.

20. Chipuk JE, Cornelius SC, Pultz NJ, Jorgensen JS, Bonham MJ, Kim SJ et al. The androgen receptor represses transforming growth factor-beta signaling through interaction with Smad3. J Biol Chem 2002; 277: 1240-1248.

21. Qi W, Gao S, Wang Z. Transcriptional regulation of the TGF-beta1 promoter by androgen receptor. Biochem J 2008; 416: 453-462.

22. Song K, Wang H, Krebs TL, Wang B, Kelley TJ, Danielpour D. DHT selectively reverses Smad3-mediated/TGF-beta-induced responses through transcriptional down-regulation of Smad3 in prostate epithelial cells. Mol Endocrinol 2010; 24: 2019-2029.

23. Jennbacken K, Tesan T, Wang W, Gustavsson H, Damber JE, Welen K. N-cadherin increases after androgen deprivation and is associated with metastasis in prostate cancer. Endocr Relat Cancer 2010; 17: 469-479.

24. Chu K, Cheng CJ, Ye X, Lee YC, Zurita AJ, Chen DT et al. Cadherin-11 promotes the metastasis of prostate cancer cells to bone. Mol Cancer Res 2008; 6 : 1259-1267.

25. Lee YC, Cheng CJ, Huang M, Bilen MA, Ye X, Navone NM et al. Androgen depletion up-regulates cadherin-11 expression in prostate cancer. $J$ Pathol 2010; 221: 68-76.
26. Kleeberger W, Bova GS, Nielsen ME, Herawi M, Chuang AY, Epstein Jl et al. Roles for the stem cell associated intermediate filament Nestin in prostate cancer migration and metastasis. Cancer Res 2007; 67: 9199-9206.

27. Gingrich JR, Barrios RJ, Kattan MW, Nahm HS, Finegold MJ, Greenberg NM. Androgenindependent prostate cancer progression in the TRAMP model. Cancer Res 1997; 57: 4687-4691.

28. Tang Y, Wang L, Goloubeva O, Khan MA, Zhang B, Hussain A. Divergent effects of castration on prostate cancer in TRAMP mice: possible implications for therapy. Clin Cancer Res 2008; 14: 2936-2943.

29. Niu Y, Altuwaiji S, Lai KP, Wu CT, Ricke WA, Messing EM et al. Androgen receptor is a tumor suppressor and proliferator in prostate cancer. Proc Natl Acad Sci USA 2008; 105: 12182-12187.

30. Thalmann GN, Anezinis PE, Chang SM, Zhau HE, Kim EE, Hopwood VL et al. Androgenindependent cancer progression and bone metastasis in the LNCaP model of human prostate cancer. Cancer Res 1994; 54: 2577-2581.

31. Sun Y, Wang BE, Leong KG, Yue P, Li L, Jhunjhunwala S et al. Androgen Deprivation Causes Epithelial-Mesenchymal Transition in the Prostate: Implications for Androgendeprivation Therapy. Cancer Res 2011; 72: 527-536.

32. Ryan CJ, Shah S, Efstathiou E, Smith MR, Taplin ME, Bubley GJ et al. Phase II study of abiraterone acetate in chemotherapy-naive metastatic castration-resistant prostate cancer displaying bone flare discordant with serologic response. Clin Cancer Res 2011; 17: 4854-4861.

33. Lin TH, Lee SO, Niu Y, Xu D, Liang $L$, Li $L$ et al. Differential androgen deprivation therapies with anti-androgens of Casodex or MDV3100 vs anti-androgen receptor of ASCJ9 lead to promote vs suppress prostate cancer metastasis. J Biol Chem 2013; 288: 19359-19369.

34. Sica A, Schioppa T, Mantovani A, Allavena P. Tumour-associated macrophages are a distinct M2 polarised population promoting tumour progression: potential targets of anticancer therapy. Eur J Cancer 2006; 42: 717-727.

35. Ueno T, Toi M, Saji H, Muta M, Bando H, Kuroi K et al. Significance of macrophage chemoattractant protein-1 in macrophage recruitment, angiogenesis, and survival in human breast cancer. Clin Cancer Res 2000; 6: 3282-3289.

36. Pienta KJ, Machiels JP, Schrijvers D, Alekseev B, Shkolnik M, Crabb SJ et al. Phase 2 study of carlumab (CNTO 888), a human monoclonal antibody against CC-chemokine ligand 2 (CCL2), in metastatic castration-resistant prostate cancer. Invest New Drugs 2012; 31: 760-768.

37. Shodeinde AL, Barton BE. Potential use of STAT3 inhibitors in targeted prostate cancer therapy: future prospects. Onco Targets Ther 2012; 5: 119-125.

cc) $\odot$ Cell Death and Disease is an open-access journal published by Nature Publishing Group. This work is licensed under a Creative Commons Attribution-NonCommercialNoDerivs 3.0 Unported License. To view a copy of this license, visit http://creativecommons.org/licenses/by-nc-nd/3.0/ 